\title{
Development of a Robotics Waiter System for the food and beverage industry
}

\author{
$\mathrm{A} \mathrm{Cheong}^{++}, \mathrm{E} \mathrm{Foo}^{+}, \mathrm{MWS} \mathrm{Lau}^{++}, \mathrm{J} \mathrm{Chen}^{+++} \& \mathrm{HY} \mathrm{Gan}^{+}$ \\ $(+$, Nanyang Polytechnic (S); ++, Newcastle University; +++, Yu Sin Engineering Work Pte Ltd (S))
}

\begin{abstract}
With significant improvement in technologies, robotics systems have appeared in homes as vacuum robots, and as transporters of goods and equipment in the industries, including hospitals and mortuaries. In Singapore (amongst others), they have attracted the attention of the food and beverage $(F \& B)$ industry, in part due to the lack of human resources here. To address the latter, a work has been established to develop the concept of robotics waiter system to serve at dining tables. It is the integration of autonomous omnidirectional platforms, a commercial indoor Radio Frequency (RF) network of sensors and transmitters and off the shelf coaster paging system commonly used in Singapore eateries. The robotics waiter system will provide services that are mundane and repetitive. The mobile platforms are designed primarily to be productive and efficient; in the current design, the torso can convey many serving trays and possibly used for cutlery collection. However unlike humanoids, they are not designed for dexterity e.g. using robotic arms and hands. Diners can order food with an iPad or tablet, and receive a coaster pager to be placed on their table. When the order is ready, the coaster pager will vibrate and activate the transmission of encoded signal (RC-5 protocol) with its table identity (id). Tables and robots are RF identification tagged and operate with the indoor RF positioning wireless network system. With proper calibration, the RF transmitters spaced at about $9 \mathrm{~m}$ apart have been tested with some success of locating tables within a meter or two. It will be evaluated in an operational F\&B outlet. The concept and design can be modified for use in the hospitals to assist in the serving of meals and medicine. With the integration of diverse commercial technologies and modular designs, it can be customized for other sectors in the service industry as well.
\end{abstract}

\section{Introduction}

It is expected that the service robots market will grow across the various continents, however, a big difference between some countries in Asia and that those in Europe and the USA is the intense level of coordination amongst the key players and the concerted effort of the governments [1] to grow that industry. This can be in part in response to their aging population and to their culture of embracing technologies. In Japan the growth of the service robotics industry will reach 65 billion yen in 2020 [2].

Robotic waiters (Figure 1) in restaurants in Bangkok, China and Japan, for example, have been featured in many online articles [3, 4]. In Singapore, due to the demographic nature and the need to moderate the dependence on foreign workers and to improve on the national productivity level, robots in the service sectors are being seriously considered.
Its first robotics waiter in the $F \& B$ Industry is the drone waiter (Figure 1), made by Singapore-based Infinium Robotics [5]. They are used to serve customers at Timbre @ The Substation, a live music bar and restaurant.
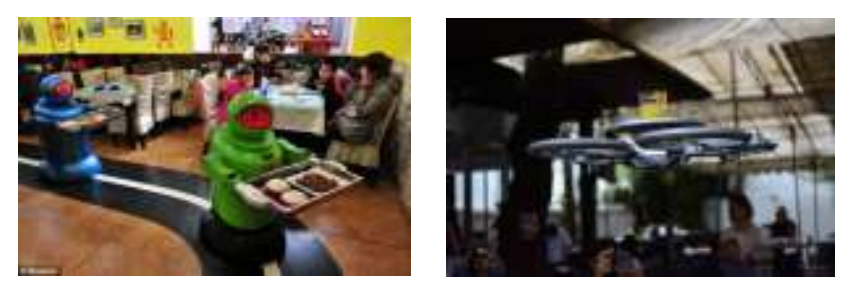

Figure 1: ground [4] and aerial robotics waiter [6]

These systems are novelties but have some limitations. The ground mobile robots navigate using paths on the ground and do not necessarily stop at the tables. The drone robot has been featured to be stable and elegant, but its payload is limited; currently it requires a waiter in attendance at the table to remove the drink or food from the hovering drone. It does not really save on manpower.

There are certain current business models in Singapore that are conducive to the introduction of robotics waiter in the industry. These models are primarily adopted to reduce the dependence on human labour. These business operations have iPad or tablets either at the dining table or at the ordering counter for diners to place their orders, and in some operations, diners are given a pager in a form of a coaster to inform the diners to collect their order when they are ready. The proposed robotics waiter system that will be presented will exploit and integrate these and other industrial systems to provide services that can reduce dependence on human labour and yet maintain a semblance of casual dining atmosphere.

\section{System Design Concept}

The design concept is based on the need to minimize human labor but not to replace it. Humans are still needed for personal relationship purpose, to engage the customers and to make their dining a pleasure. For all the numerous research on intelligent autonomous robots, it remains a difficult problem for such robots to work in a real world environment of a restaurant. A more viable approach is the integration of the autonomous robots within a smart environment [7].

Hence the mechanical waiter robot is not designed to be a humanoid, with its present limitations in the maneuverability. Therefore a wheeled mobile base will be productive, efficient and hygienic. It will be designed to be 
maneuverable, with a low level computing for basic manoeuvrability and safety behaviours; the eco-system has the higher level computer for the main functions and for communication with the indoor location network computer.

\section{A. The Eco-system Description}

The robotics system is a total eco-system. It does not have a truly intelligent robot, but an autonomous omnidirectional waiter robot integrated with the food ordering system (Central System), the indoor location system and a table identification and locating system. Figure 2 illustrates the system architecture of the waiter robot system. The centralised computing handles components such as sensors, camera, speakers, battery charging system, the lower level of computing (base of the robot) and the order delivery system.

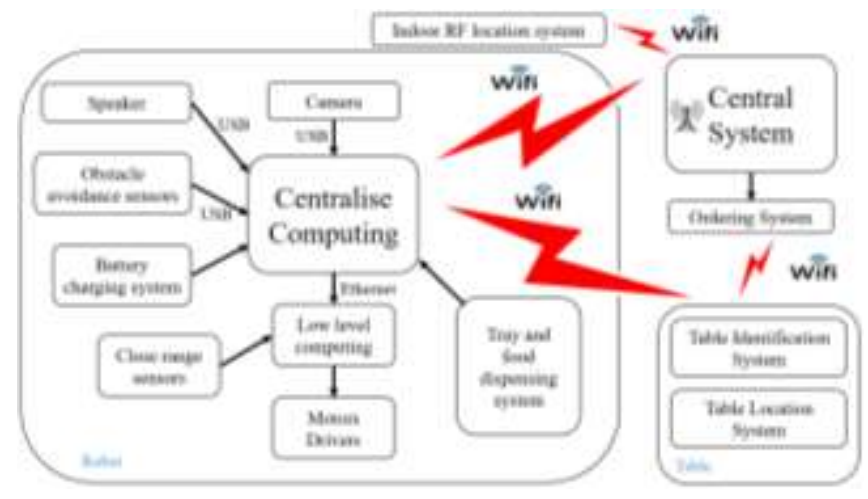

Figure 2: System Architecture

Each table has an embedded processor, infrared (IR) transmitter and a radio-frequency identification (RFID) tag with a unique code which allows the system to know where a patron is seated. Using the tablets either on the tables or on the waiter robot, patrons place their orders through the central ordering system and will be issued with a coaster pager (description given below) which is coded with the patrons' order number. When the items are ready, the pager is activated (as is currently the case in some places) and the table RFID code is transmitted which allows the indoor RF location system to identify the location of where the food is to be delivered. The food is loaded into the robot which then delivers the items to the table where the coaster pager is located.

\section{B. Waiter Robot}

The waiter robot is designed in three main parts: the base, the torso and the sensing system as shown in Figure 3.

The torso of the robot stores the ordered items to be delivered. It keeps the food unexposed to the environment and is served to the diners at their table. The torso is able to house multiple levels of trays. It is designed like the dummy waiter in some restaurants. Each tray can be brought up to the door level and be dispensed onto the table when the door is lowered. It is possible to have serving doors on either side of the torso so that the robot movements are minimised. The service door can be automatically adjusted, using a scissor lifting mechanism, to different table heights in different restaurants.

The base of the robot houses the motors and drive systems that propel the robot. In order that the robot is able to navigate in tight and constrained spaces typical of most casual dining outlets in Singapore, it is designed as an omnidirectional vehicle with mecanum wheels. This means that the robot can move side wards or move in an angle without changing the robot orientation $[8,9]$. Hence besides being manoeuvrable, it is also economical in its movements.

The robot navigates using waypoints from its planner and has the capability to avoid obstacles dynamically using laser sensors. It has IR sensors to read the RC-5 protocol that is transmitted from the table location and identification system and sensors to detect how close it is to the table below lowering its service door to deliver the trays containing the food.

\section{Table location and Identification}

This is a critical part of the robot locating the table and delivering its load. It is conceived in-house integrating a commercial coaster pager, an embedded processor, an IR transmitter and the table RFID with a unique table code. Each table will be installed with it. Each table has a unique RFID code that is locatable by the indoor RF location system, the 1RWave.

The patron inserts the coaster pager that contains his paging code for the food ordered into the system at the table. When the patrons' food are ready, the pager will vibrates and activates the embedded processor to instruct the IR transmitter to transmit the table RFID code using the RC-5 protocol system found in remoter controllers for home use.

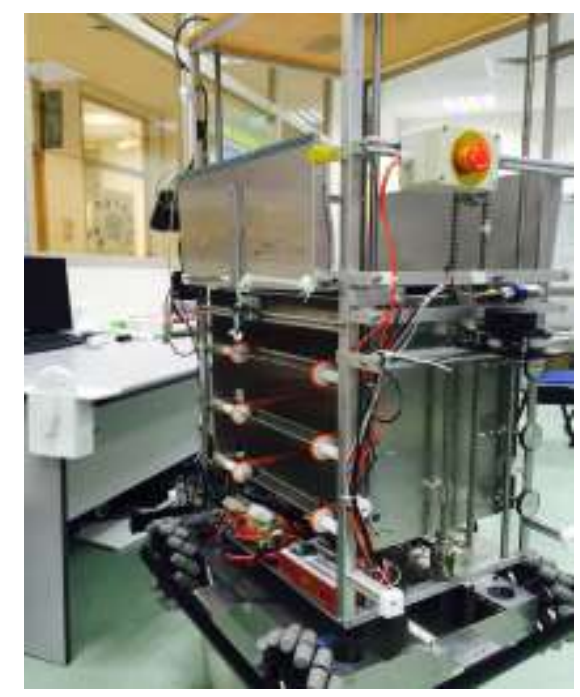

Figure 3: Waiter robot design and the table location system

The table id is read with the help of the indoor RF location system and the robot is "informed" of the table location. In 
addition, as the robot approaches the zone in which the table is located, its IR receiver too will pick up the RC-5 protocol of the table id. The robot will then use its pair of IR sensors on its side to align the robot as much as possible with the robot before delivering the food.

\section{Software Implementation}

A Robot Operating System (ROS) is a collection of software frameworks that is available online. It provides basic functionalities and many packages that have been developed and are ready to use, allowing the designers to focus on their specific requirements, and modifying them according to their needs. Although ROS is not a real time operating system, it consists of standard operating system services such as device control which is needed in the functionality of the robot.

The robot computer uses Linux OS which hosts the ROS on board. Windows OS and Linux can communicate using the ROS-NET and ROS-BRIDGE package. A map can be preloaded into the robot using the map server package and controlled through the ROS-RVIZ package.

There are different layers of drivers and control algorithm designed for the waiter robot. Three micro-controllers (mbed) are used to provide communication and control over the base, the tray delivery system and the attitude heading reference system (AHRS). Mbed controller has a powerful microchip and also and open source $\mathrm{C}++$ platform. These micro-controllers will be communicated to a Next Unit Computing (NUC), a Linux computer with ROS running on it. A central system with the order system, 1RWave and the radio frequency (RF) location system communicates with the robot through Wireless Fidelity (WiFi) Network. Figure 2 illustrates the control architecture proposed for the waiter robot.

\section{Iv. Navigation System}

An autonomous robot requires a technique to navigate and locate itself. Path planning, Odometry, the AHRS and the $1 \mathrm{RW}$ ave indoor RF location system are implemented to as a navigation strategy for the waiter robot.

\section{A. Navigation and Path Planning}

The floor plan of the restaurants is usually available. This can be used to provide a map for path planning with appropriate unique features used as way points for path planning - the robot navigates according to the path generated for each task or order. It is possible also to locate RFID tags at some way points. The robot uses a combination of dead reckoning Odometry, dedicated localization on certain waypoints and the use of AHRS to find its orientation and bearing for the robot to navigate.

Alternatively, Simultaneous Localisation and Mapping (SLAM) can be used for the navigation of the robot $[10,11]$.
It is a well-established tool that a robot uses to generate a map on the move and then locates itself on the map it creates. Such method can be accurate and useful but with patrons moving table and relocating themselves, the computer map may not be useful.

\section{B. Odometry}

The positions of the robot can be computed by calculating the robot's position relative to the initial location using encoders. The distance travelled by the robot will be known by using the Odometry technique. However, such technique is known to have errors that can accumulate over distance. In this application, the Odometry values are corrected on certain waypoints to compensate possible slip and miscalculation while the robot navigates.

\section{Altitude Heading Reference System - heading of the robot}

The orientation of the robot is given by the on board AHRS. It is common sensor use in aeroplanes and robots now to give the heading and the other attitude angles. The sensor provides a 9 degree of freedom with drift correction, which is applied to counter the gyroscope's drift over time. The readings of the AHRS are used to match with various know orientations obtained from the floor plan of the restaurant. Therefore, with the known orientation, the robot is able to turn to the specific bearing or direction by using the compass and maintains its orientation while navigating.

\section{Light detection and ranging}

The "eyes" of the robot is a pair of Lidar (light detection and ranging) located front and back of the waiter robot. The Lidars emits a low power light source at steps of $1^{\circ}$ over $360^{\circ}$. The reflected light rays are captured and processed to give the range of objects at increment of $1^{\circ}$. Such data can be used (a) to indicate free space for the robot to move; (b) detect objects that requires either the robot to stop or to avoid and (c) to create a computer map of the environment using SLAM software.

\section{E. Indoor RF location System: 1RWave}

The table location system is based on active RFID, 1RWave system as shown in Figure 4 connected in mesh network. The implementation of the 1Rwave technology will allow the identification of the robot's position at real time. It also allows the robot to locate the customer's position and hence uses its path planner to move from the serving point to the right table. The system consists of readers, tags and coordinators. It uses at least three readers or coordinator combinations to locate the position of the tags. The positions of the tags are usually updated at $5 \mathrm{~Hz}$. However, as we need to track the positions of the tables and the robot, the update 
has to be reduced to $1 \mathrm{~Hz}$. The advantage of this system is that it is connected via a mesh network and is able to recover and provide the location information when one of the active readers is down.

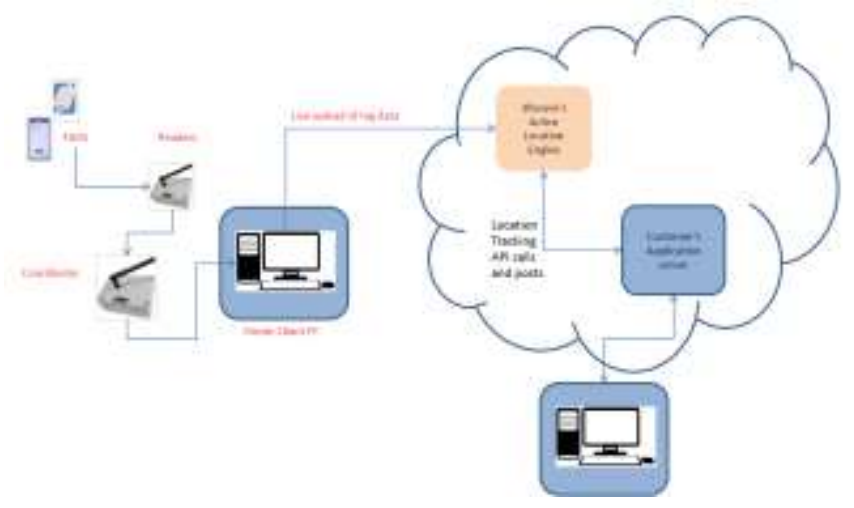

Figure 4: 1R wave active RFID system

Another advantage of this system is that the computation of the location is hosted at the cloud thus making it easy to access the location information from anywhere. At this moment the system is able to give an accuracy of 1 meter.

\section{v. Evaluation \& Discussion}

Development of the system requires testing and evaluation of the viability of the concepts being implemented. This can be done initially with prototypes before full scale investment.

\section{A. Prototype - the robot base}

A low cost prototype was used to develop the motion control algorithms and the base computer communication with the robot main computer.
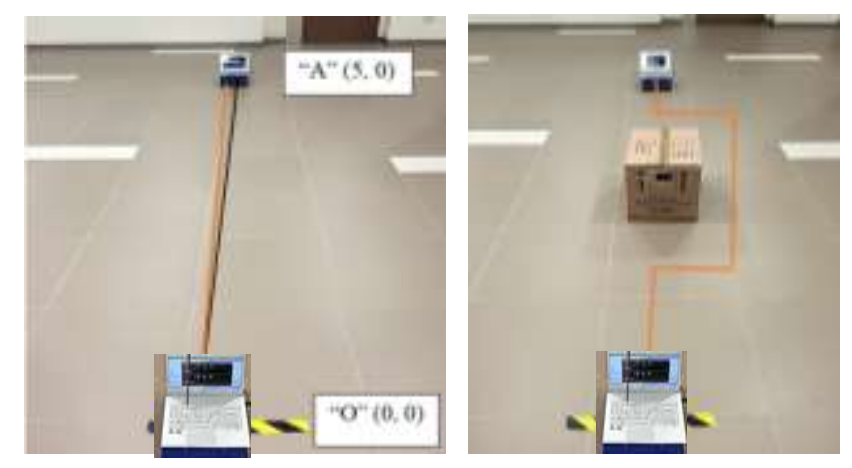

Figure 5: base prototype under test

The estimation of the encoders for Odometry use and the avoidance of obstacles were tested. Figure 5 shows there is a side ward drift which had to be corrected using the AHRS to provide a closed loop heading control.

\section{B. Delivery System - getting closer}

The robot being directed to about a metre or two from the target table has to be navigated closer to the table. The concept and the algorithm described above had been evaluated using the prototype and will tested on the full scale waiter robot in the restaurant. Here two sets of IR sensors are used on the robot: long range and short range.
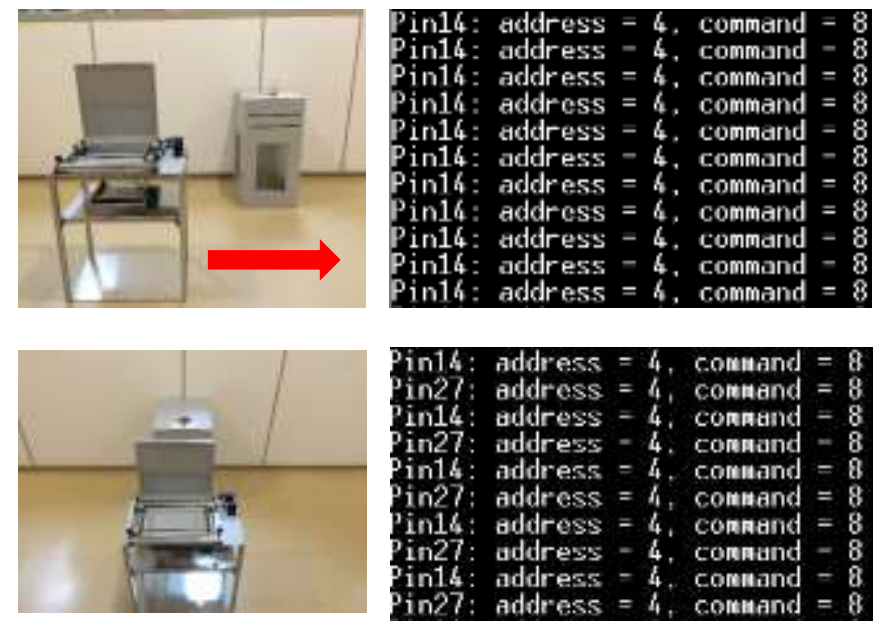

Figure 6: testing of the receipt of the Table id by robot

As the robot approaches the target table (Figure 6), its transmitted id is captured by the robot (Pin 14, long range IR sensor, reading the id as command). As it draws closer, the robot short range captures the id (Pin 27). Auto-correction of robot positions are still being developed for finer docking of robot to table.

\section{Trial tests at the restaurant}

Trials in a restaurant have shown that it is feasible to operate the proposed waiter robot system in a restaurant environment. However, it has been observed that the mechanical structure of the robot, and the indoor positioning system features have to be improved in the subsequent development of the robot.

The vibration issue caused by the robot during navigation has to be minimised. The problem causing the vibration comes from the mecanum base structure of the robot. It is noticed that drinks are spilled during the motion. The addition of damping system has to be incorporated.

The 1RWave system takes some time to settle and as a result it can be difficult to track the robot on the move. It is also realised that the signal bounces across the uneven ceilings and results in data uncertainty. There is a need to locate the robot using RFID at strategic way points.

\section{vi. Safety}

The safety aspect of the system has been carefully drafted and designed. Hence the robot moves at about $0.3 \mathrm{~m} / \mathrm{s}$ and 
slows further down when the front Lidar detects an object, and comes to stop if it is very close to the object.

In addition, safety bumpers connected to hard switches will trigger a robot stop motion when any of the bumpers come into contact with an object.

A "kill" knob, prominently located at the front of the robot, when activated, will break power supply to all motors and the system.

On approaching the table, there are sensors on the door and sides of the robots to prevent the service door from being lowered if there objects detected or being raised when the tray is still on the door.

As the robot is powered by lithium polymer (LiPo) battery, it is important to ensure the battery is not over use by using the battery alert system and if the robot over power by drawing a high level of current, the fuse will cut its supply to prevent further damages to the system.

\section{vII. Conclusion}

The robotic waiter system is a design concept that integrates autonomous omnidirectional mobile robots with an ecosystem which provides knowledge of the environment in which the robots operate. This allows for a quicker translation from research to the industry. The robots are designed to be productive and efficient replacement where there is a shortage of human labour which does mundane and repetitive work such as carrying food to the tables. In addition, the ability to carry more than one order in the robot significantly helped in reducing the workload of a waiter during peak periods. Tests with the prototype and full scale robot in the restaurant have shown that the design is able to provide assistance in the restaurant, and therefore the next challenge is to have multiple robots in the restaurant. With a one off development of the smart eco-system, the cost of additional autonomous robots will increase the total cost significantly as compared with using multiple state of the art AI waiter robots with human skills and abilities.

\section{Acknowledgment}

This project is funded by internal funding of the School of Engineering, Nanyang Polytechnic, Singapore, funding BH150740 of the Newcastle University International Singapore, and the Tech Factor Challenge 2015 Organizers, Singapore

The technical support provided by Mr Noel Teo is greatly appreciated.

\section{References}

[1] Kumar, Vijay, George Bekey, and Yuan Zheng. Industrial, Personal And Service Robots. 2015. Print. WTEC Report: Review Of U.S. Research In Robotics, Chapter 5, 2006.
[2] Myoken, Yumiko. 'Japan'S Service Robotics Development'. Science \& Innovation Section, British Embassy Tokyo, 2011 (2011): n. pag. Print.

[3] Mail Online,. 'Mechanic Masterchef: Robots Cook Dumplings, Noodles And Wait Tables At Restaurant In China'. N.p., 2013. http://www.dailymail.co.uk/news/article-2261767/Robot-RestaurantRobots-cook-food-wait-tables-Harbin.html\#ixzz3kfVgSdb7. 4 Sept. 2015.

[4] Wilson, Allan. 'Hajime Robot Restaurant, Bangkok, Japanese Yakiniku Barbecue'. N.p., 2015. http://live-less-ordinary.com/hajimerobot-restaurant-in-bangkok-yakiniku/. 3 Sept. 2015.

[5] Welcome to Infinium Robotics,. 'Intelligent Robotics Solutions Infinium Robotics'. N.p., 2015. http://www.infiniumrobotics.com/. 3 Sept. 2015.

[6] Tech in Asia,. 'Singapore Restaurant Shows Off Autonomous Drone Waiters'. N.p., 2015. https://www.techinasia.com/singaporerestaurant-autonomous-drone-waiters/. 3 Sept. 2015.

[7] Park, Jae-Han, Seung-Ho Baeg, and Moon-Hong Baeg. ' An Intelligent Navigation Method For Service Robots In A Smart Environment'. International Conference on Control, Automation and Systems 2007 (2007): n. pag. Print.

[8] Lin, L. and Shih, H. (2013). Modelling and Adaptive Control of an Omni-Mecanum-Wheeled Robot. ICA, 04(02), pp.166-179.

[9] McInerney, I. (2015). Simplistic Control of Mecanum Drive. FRC Team 2022, pp. 1 - 4

[10] Kuo, Bor-Woei et al. 'A Light-And-Fast SLAM Algorithm For Robots In Indoor Environments Using Line Segment Map'. Journal of Robotics 2011 (2011): 1-12. Web.

[11] Pei, Fujun, Mei Wu, and Simin Zhang. 'Distributed SLAM Using Improved Particle Filter For Mobile Robot Localization'. The Scientific World Journal 2014 (2014): 1-10. Web.

About Author (s):

Mr Aaron Cheong is currently a Postgraduate student with Newcastle University, UK. He is also a Newcastle University Alumnus

Dr Edwin Foo is an academic staff with the School of Engineering, Nanyang Polytechnic, Singapore and also an adjunct staff Newcastle University

Dr Michael W S Lau is an academic staff with the School of Mechanical and Systems Engineering, Newcastle University based in Singapore

Mr Josh Chen Ye Seng is currently with Yu Sin Engineering Work Pte Ltd, and an Alumnus of both Newcastle University and Nanyang Polytechnic

Mr Gan Hsien Yan is currently a student with the School of Computer Engineering, Nanyang Technological University (S), and a Nanyang Polytechnic Alumnus 